\title{
DisEÑO, CONSTRUCCIÓN E IMPLEMENTACIÓN DE UNA PLATAFORMA ROBÓTICA MULTIFUNCIONAL CON PROPÓSITOS DIDÁCTICOS DINGO 1.0
}

\author{
Henry Cubides ${ }^{1}$, León Cuvi $^{1}$, José Luis Cuzco ${ }^{1}$, Esteban Ordoñez ${ }^{2 * *}$
}

\section{Resumen}

El presente proyecto presenta el diseño y construcción de una plataforma robótica mediante el uso de elementos y dispositivos que se encuentran fácilmente en el mercado, tratando siempre de optimizar al máximo la economía del grupo de trabajo. Se empieza por definir parámetros mecánicos para el correcto funcionamiento de la oruga ya sea para terrenos hostiles, gradas, etc., de igual forma partes electrónicas circuiterías y controladores. Con el propósito de que la plataforma sea lo más fácil y atractiva de manipular para el usuario, se implementó una variada gama de controles mecánicos eléctricos y de software, empleando comunicación inalámbrica para facilitar la mobilidad. El producto final presentado es una plataforma con distintas formas de control inalámbrico y de aplicaciones variadas para el usuario. Se detallan a continuación los procedimientos técnicos y físicos para la construcción, diseño y uso del producto Dingo 1.0, ya sea para conocer sus partes físicas o simplemente para programar la plataforma.

Palabras clave: EVA, comunicación, controladores, plataforma educativa

\begin{abstract}
This work presents the design and construction of a robotic platform using elements and devices that are easily found on the market, always trying to optimize the economy of the workgroup. It begins by defining mechanical parameters for the proper functioning of track either hostile terrain, stairs, etc., and the electronics circuitry and drivers. With the purpose of developing an easy and attractive way to manipulate the platform, we implemented a wide range of electrical, mechanical and software as controls, using wireless communication to facilitate mobility. The final product presented is a platform with various forms of wireless control and various user applications. Detailed below are technical and physical procedures for construction, design and use of Dingo 1.0 product, either to meet their physical parts or simply to set the platform.
\end{abstract}

Keywords: EVA, communication, controllers, educative platform

${ }^{1}$ Estudiante de Ingeniería Eléctrica de la Universidad Politécnica Salesiana Sede Cuenca.

${ }^{2, *}$ Ingeniero Eléctrico, Máster en Desarrollo del Pensamiento, Docente de la Universidad Politécnica Salesiana, Estudiante de la Maestria Gestion de Telecomunicaciones, Universidad Politecnica Salesiana. Autor para correspondencia: eordonez@ups.edu.ec

Recibido: 16 - Marzo - 2012; Aprobado tras revisión: 25 - Mayo - 2012

Forma sugerida de citación: Cubides, H. Cuvi, L. Cuzco, J. y Ordoñez E. (2012). "Diseño, construcción e implementación de una plataforma robótica multifuncional con propósitos didácticos DINGO 1.0". INGENIUS. N7, (enero/junio). pp 29-34. ISSN: 1390-650X 


\section{Introducción}

En la actualidad los sistemas robóticos forman parte de la vida cotidiana del ser humano, no solo la gran mayoría de industrias trabajan con robótica, los robots también son parte de equipos de rescate o son creados simplemente para nuestro entretenimiento. Si bien el desarrollo de este tipo de sistemas generan un beneficio, su propósito principal es la de despertar un interés de manera que se eliminen ciertas barreras que existen en la sociedad.

Razón por la cual vemos la necesidad de implementar una plataforma robótica multifuncional, con la finalidad de presentar un producto de multitareas que beneficie a instituciones educativas, privadas y público en general.

\section{Generalidades de los robots}

Un robot es un agente artificial mecánico o virtual [1]. También se puede decir que es una máquina usada para realizar un trabajo automáticamente y que es controlada por una computadora [2]. Por otro lado un robot está diseñado para sentir y manipular su entorno mediante sensores de percepción y actuadores.

Tiene cierta inteligencia o habilidad para tomar decisiones basadas en el ambiente o en una secuencia reprogramada automática. Puede moverse o desplazarse en uno o más ejes de rotación o traslación. La Tabla 1 resume las características principales de los robots, los tipos existentes y su aplicación.

\section{A. Los robots didácticos o experimentales}

Los robots didácticos están dedicados a la enseñanza y aprendizaje de la robótica, y típicamente no cumplen una tarea específica como tal. Generalmente tienen la forma de un brazo mecánico que imita la forma humana o replica la geometría de los robots industriales.

Básicamente se puede decir que hay dos tipos de robots didácticos: los estáticos, que
Tabla 1. Generalidades de los robots

\begin{tabular}{ccc}
\hline Características & Tipos & Aplicaciones \\
\hline Movilidad & Industriales & Todos \\
\hline Gobernabilidad & De servicio & Todos \\
\hline Autonomía & Inteligentes & Todos \\
\hline Polivalencia & Humanoides & Todos \\
\hline Programable & Educativos & Todos \\
\hline
\end{tabular}

van sobre una base fija, y los móviles, que van montados sobre una plataforma que se puede desplazar sobre una superficie [3].

\section{B. Plataformas educativas}

Existen numerosas investigaciones y aplicaciones en el campo educativo, creación de modelos para el aprendizaje; utilización de multimedios, telemática, teleinformática, tele enseñanza, y ahora, algoritmos genéticos y realidad virtual [3]. El desarrollo de estas plataformas de tipo educativas permite la formación científica y tecnológica, la expresión de emociones y sentimientos por parte de los usuarios de estas tecnologías y estimula el desarrollo cognitivo de los estudiantes. En la Tabla 2 se muestra los sistemas que constituyen una plataforma robótica.

Tabla 2. Sistemas de una plataforma robótica

\begin{tabular}{ll}
\hline Sistema mecánico & $\begin{array}{l}\text { Mecanismos, actuadores y tornillos, } \\
\text { así como el equipo robótico comple- } \\
\text { mentario, el cual permite la correcta } \\
\text { realización de las tareas requeridas. }\end{array}$ \\
\hline $\begin{array}{l}\text { Sistema de percep- } \\
\text { ción }\end{array}$ & $\begin{array}{l}\text { Transductores y circuitos electróni- } \\
\text { cos asociados que permiten la gen- } \\
\text { eración de señales eléctricas para } \\
\text { mostrar el estado de su entorno. }\end{array}$ \\
\hline Sistema de control & $\begin{array}{l}\text { Constituido por uno o varios proce- } \\
\text { sadores para interactuar con los } \\
\text { otros sistemas. }\end{array}$ \\
\hline $\begin{array}{l}\text { Sistema de comu- } \\
\text { nicación hombre- } \\
\text { maquina }\end{array}$ & $\begin{array}{l}\text { Permite al usuario la comunicación } \\
\text { con el robot para darle las instruc- } \\
\text { ciones que conforman tareas especí- } \\
\text { ficas. }\end{array}$ \\
\hline
\end{tabular}

\section{Construcción de la plataforma robótica DINGO 1.0}

Todo proyecto inicia con un diseño. En el presente proyecto se ha considerado los cuatro 
sistemas principales detallados anteriormente: el sistema mecánico conformado por la estructura, locomoción, engranajes, transmisión de torque y fuerza. El sistema de percepción, conformado por el mando de los motores, sensores y transductores. El sistema de control para el accionamiento y procesamiento de cada una de las señales provenientes de la parte eléctrica. El sistema de comunicación hombre/máquina, encargada del monitoreo, proporcionará además una interfaz gráfica amigable.

\section{A. Sistema mecánico}

Para la construcción se empieza diseñando la estructura base en la cual se soportará todo el peso de motores, circuitería y aplicaciones; cabe notar que el diseño se lo realizó de forma estructurada de tal manera que en el proceso de ensamblaje se puedan realizar las debidas pruebas.

Se debe tener especial cuidado en la longitud de las cadenas, dado que un ajuste excesivo puede ocasionar que la cadena se rompa, o al contrario una cadena floja puede librarse fácilmente.

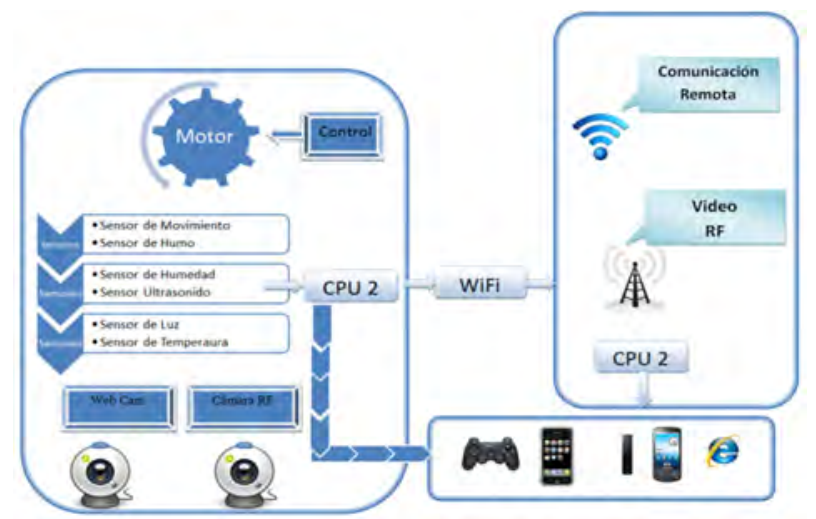

Figura 1. Diagrama de bloques plataforma DINGO 1.0.

Las orugas se construyen mediante una cadena que rodea las llantas, delanteras y traseras, cuyo fin es aumentar la superficie de contacto con el suelo y conseguir una mayor tracción. Las orugas permiten rebasar mayores obstáculos que solamente usando ruedas e incluso subir escaleras. Como desventaja se presenta la gran cantidad de energía que necesita el robot en los giros. El giro se realiza rotando las cadenas en sentidos opuestos, de modo que las fuerzas inversas hacen girar el robot.

Para el movimiento de las orugas se utilizó motores para pluma de camión los cuales pueden trabajar a $12 / 24 \mathrm{~V}$. Se trata de un sistema de fuerza centra que consta de una caja de engranes la cual posee un tornillo sin fin y un piñón. La última etapa es la de pintura. Para esto es necesario pulir asperezas y lijar los detalles que no están de acuerdo a lo deseado. Con esto se logra también evitar la corrosión y brindar a la estructura un toque elegante.

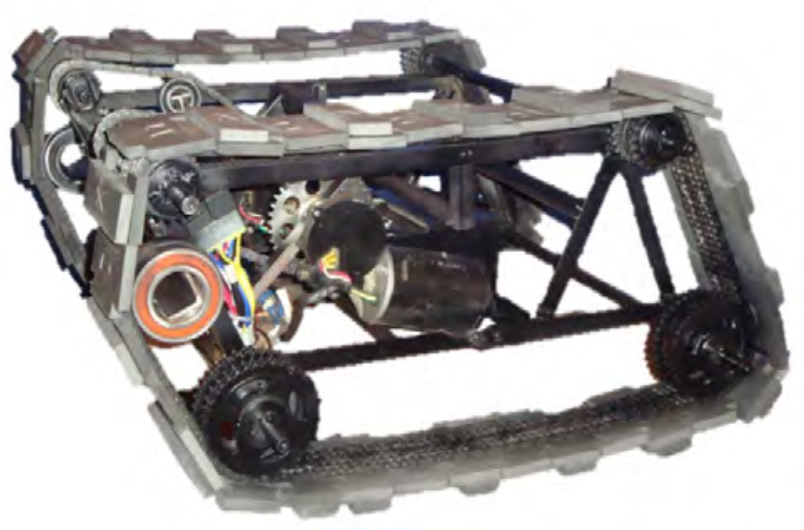

Figura 2. Estructura mecánica plataforma DINGO 1.0.

La Figura 2 muestra el sistema mecánico completo de la plataforma robótica, el chasis ha sido construido con perfil estructural y varillas de hierro, la oruga está conformada por piñones y cadenas dobles que permiten el desplazamiento de la misma, recubiertas con EVA, un material similar al caucho pero con mejores prestaciones, ganando mayor agarre en escalones y en superficies lisas.

\section{B. Sistema de percepción}

Los sensores que integran esta plataforma son: dos de temperatura, uno para la parte exterior y otro para controlar la temperatura interna del mismo; un sensor para la detectar la cantidad de iluminación, un sensor de movimiento y un sensor de ultrasonido. Todos estos son procesados por separado de 


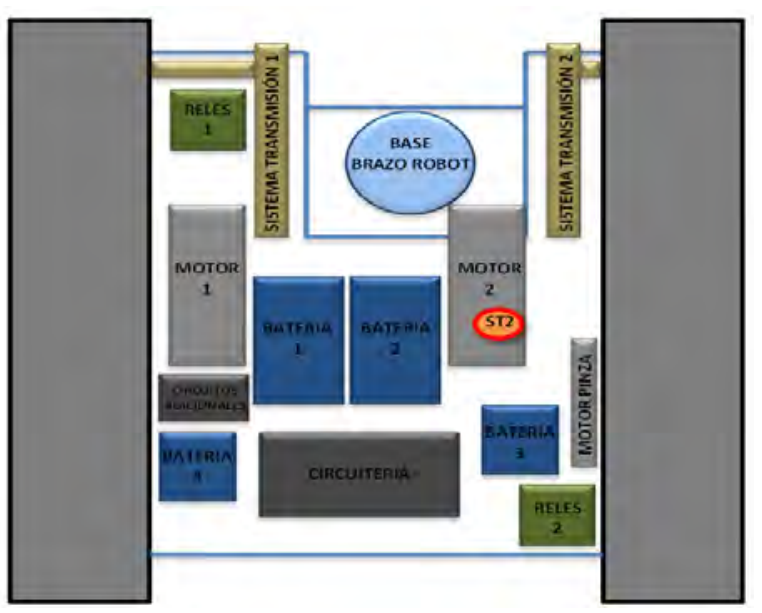

Figura 3. Distribución de la parte interna del DINGO 1.0 .

modo que sea posible identificarlos con facilidad para futuras pruebas de control y mantenimiento.

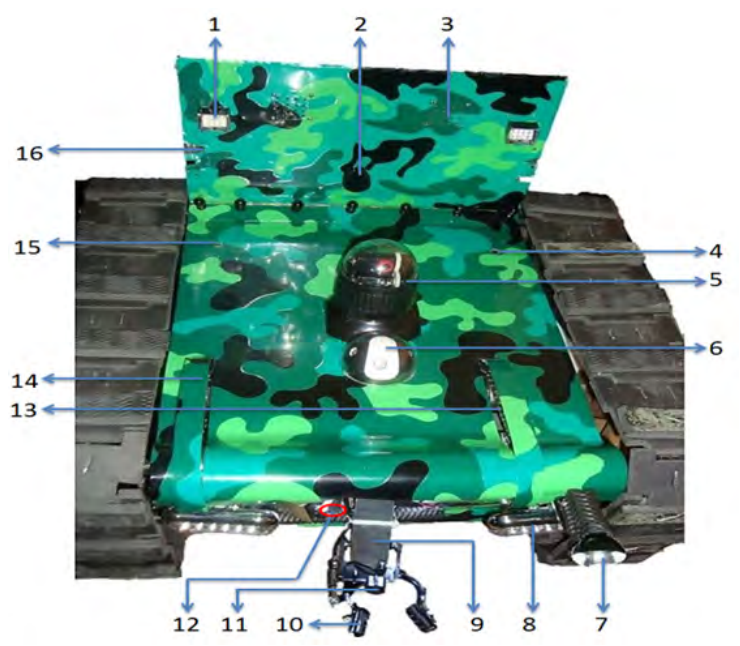

Figura 4. Ubicación de los sensores y actuadores.

\section{Sistema de control}

La programación se desarrolló en PicBasic, versión 2.2.1.1. Los motores han sido conectados directamente a los puertos para su funcionamiento. Se tiene dos microcontroladores 18F4550, uno se encarga de la comunicación USB con el computador y el segundo para el control de los dispositivos implementados, los sensores, los servos, entre otros. Para el aislamiento entre la etapa de control y la de potencia se utilizaron drivers, TIP's y relés Bosh
Tabla 4. Detalles sistema de percepción DINGO 1.0

\begin{tabular}{cc}
\hline$\#$ & Dispositivo \\
\hline 1 & Luces posteriores \\
\hline 2 & Sensor de Humo \\
\hline 3 & Sistema de Ventilación \\
\hline 4 & Sensor de Humedad \\
\hline 5 & Webcam (cámara principal) \\
\hline 6 & Sensor de Luz \\
\hline 7 & Sensor Ultrasonido (Radar) \\
\hline 8 & Luces frontales \\
\hline 9 & Brazo Robótico \\
\hline 10 & Pinza \\
\hline 11 & Cámara RF \\
\hline 12 & Chicharra \\
\hline 13 & Motor 1 \\
\hline 14 & Motor 2 \\
\hline 15 & Sensor de Temperatura 1 \\
\hline 16 & Sensor de Movimiento \\
\hline
\end{tabular}

de $30-40$ A.

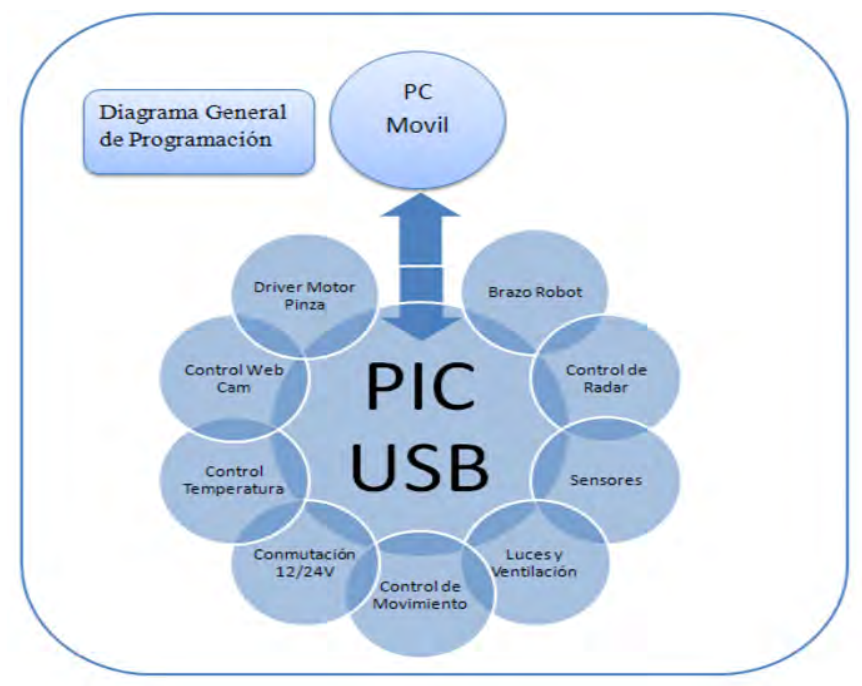

Figura 5. Diagrama general de programación.

Físicamente cada sistema es modular, es decir, está diseñado para trabajar por separado, con controladores independientes, facilitando así su manipulación.

\section{Sistema de comunicación hombre/máquina}

El robot puede guiarse y manipularse mediante un control de PS3, un control de Nintendo Wi, un celular con Wi-Fi vía internet, vía radar, con aplicaciones en ipad, ipod y 
Cubides et al / Diseño, construcción e implementación de una plataforma robótica

Tabla 3. Generalidades de los robots

\begin{tabular}{ccl}
\hline Elemento & Alimentación & Detalles \\
\hline Bateria 1 & $12 V c c$ & Alimentación motor 1 \\
\hline Bateria 2 & $12 V c c$ & Alimentación motor 2 \\
\hline Bateria 3 & $12 V c c$ & Alimentación motor 3 \\
\hline Bateria 4 & $12 V c c$ & Alimentación circuitería + sistema de iluminación \\
\hline Motor 1 & $12 V c c-24 V c c$ & Motor de marcha Izquierdo \\
\hline Motor 2 & $12 V c c-24 V c c$ & Motor de marcha Derecho \\
\hline Motor pinza & $12 V c c$ & Motor para accionar pinza \\
\hline Sistema de transmisión 1 & Cadenas & Transmisión de movimiento motor 1 \\
\hline Sistema de transmisión 2 & Cadenas & Transmisión de movimiento motor 2 \\
\hline Relé 1 & $30 A-40 A$ & Relés de marcha motores 1 y 2 (Puente H) \\
\hline Relé 2 & $30 A-40 A$ & Relés de conmutación de alimentación $(12 V / 24 V)$ \\
\hline Base brazo robot & & Motores 1 y 2 \\
\hline Circuiteria & $12 V c c$ & Ubicación Brazo Robótico \\
\hline Circuitos adicionales & $30 A-40 A$ & Humedad, Iluminación, Luces y Ventilación. \\
\hline ST2 & $5 V c c$ & Sensor de Temperatura 2 \\
\hline
\end{tabular}

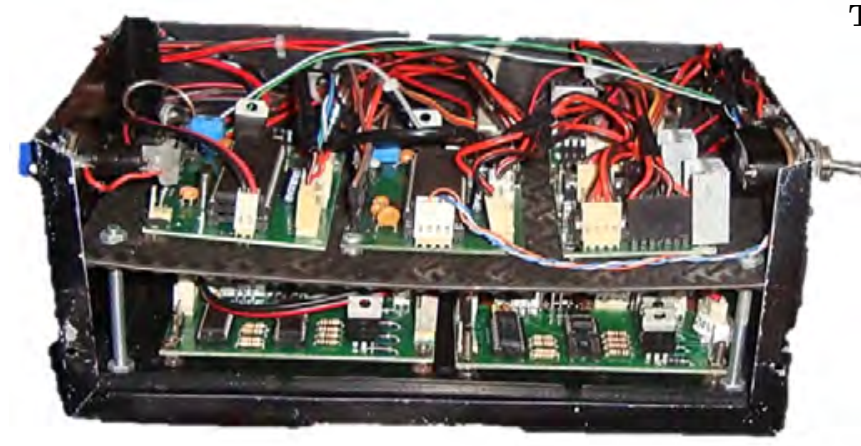

Figura 6. Caja de controladores.

en general con dispositivos que cuenten con tecnologías soportadas. El software utilizado para la adquisición, manipulación y monitoreo de la plataforma ha sido elaborado en el programa LABVIEW de National Instruments; además, para esta aplicación se han elaborado 2 tipos de programas, cada uno de los cuales posee diferentes funciones, mismas que se describen a continuación.

\section{Panel fijo}

El programa principal o también denominado "programa fijo", posee todas las funciones con las que ha sido diseñado el robot. Esta ventana proporciona una variedad de controles e indicadores que per-
Tabla 5. Componentes caja de cantroladores DINGO 1.0

\begin{tabular}{cc}
\hline$\#$ & PLACAS \\
\hline PL1 & Controlador Brazo Robótico \\
\hline PL2 & Controlador Principal y Comunicación \\
\hline PL3 & Controlador Chicharra \\
\hline PL4 & Controlador Radar \\
\hline PL5 & Controlador Webcam \\
\hline PL6 & Driver Motor Pinza \\
\hline PL7 & Controlador Temperatura \\
\hline PL8 & Driver Reles Marcha (Puente H) \\
\hline PL9 & Driver Relés conmutación $12 / 24 \mathrm{~V}$ \\
\hline
\end{tabular}

miten al usuario manipular la plataforma de acuerdo a lo que él requiera. La ventana principal contiene varias etapas, las cuales se ilustran en la siguiente Figura 7 .

- Etapa de movimiento

- Etapa de indicadores

- Etapa de visión

- Etapa de botones

- Etapa de brazo robot

- Etapa de sonar

- Etapa de selección de equipo

- Etapa de secuencias 


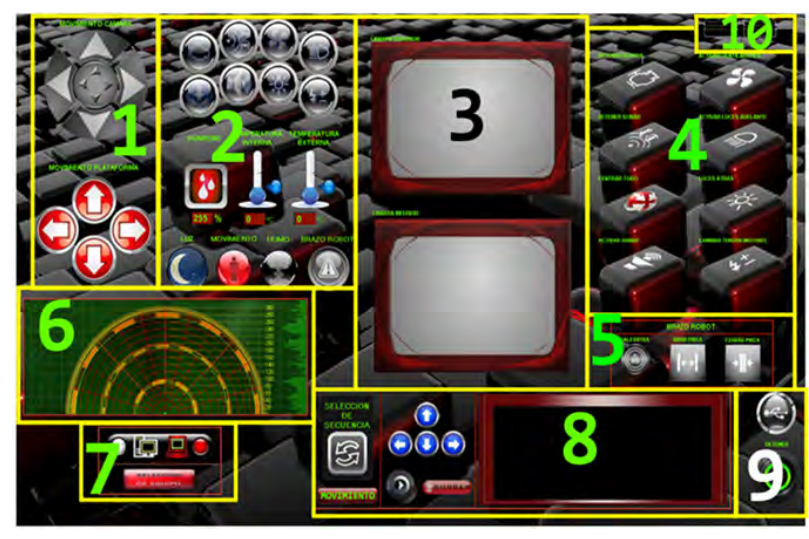

Figura 7. Panel fijo DINGO 1.0.

- Etapa de estado de conexión del robot y apagado

- Etapa de estado de baterías

\section{Panel móvil}

El programa o panel móvil es aquel donde el usuario puede acceder desde un dispositivo móvil, como una tableta o un teléfono celular que lo soporte a través de una página web. Para poder controlar a la plataforma, al igual que el programa fijo, este está compuesto por una variedad de etapas, como se muestra en la Figura 8.

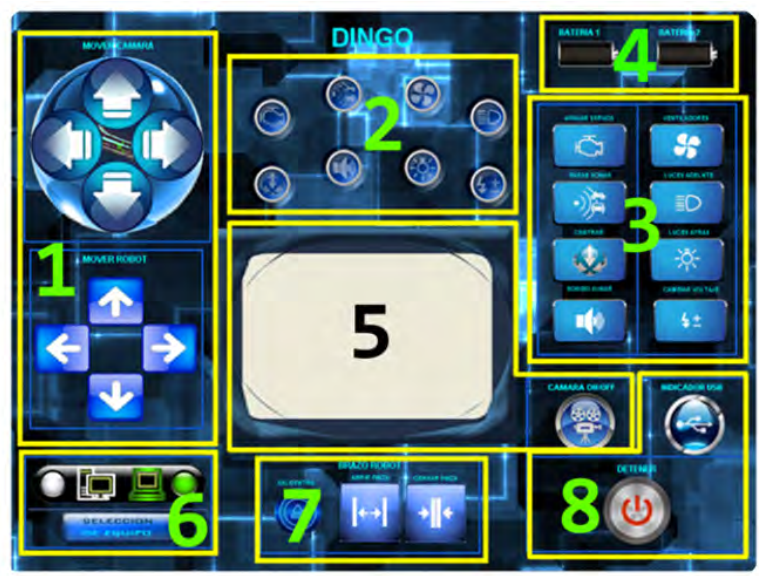

Figura 8. Panel móvil DINGO 1.0.

- Etapa de movimiento

- Etapa de indicadores de funciones

- Etapa de botonera de funciones
- Etapa de estado de baterías

- Etapa de visión

- Etapa de selección de equipo

- Etapa de brazo robot

- Etapa de estado de conexión del robot y apagado

\section{Conclusiones}

Las posibilidades de explotación de un proyecto de armado, diseño y construcción de prototipos didácticos son múltiples. Tocan conceptos y conocimiento sobre principios de ingeniería mecánica, física, electricidad, electrónica, informática e incluso la posibilidad de hacer "inteligente" el programa que controla el dispositivo robótico desde el punto de vista lógico y computacional.

La idea de esta plataforma es dar al operador ya sea programador, estudiante, investigador o a quien le guste interactuar con dispositivos electrónicos y mecánicos, la posibilidad de manipular a su antojo el aparato robótico, fomentándose un proceso de aprendizaje de una manera más didáctica, amigable y productiva.

Se ha demostrado que es posible la elaboración de una plataforma multifuncional con las herramientas y elementos que el grupo de trabajo puedo conseguir en el mercado, lo que pone de manifiesto que un proyecto de estas proporciones puede autofinanciarse, lo que a su vez resalta las habilidades de diseño, planificación, producción y programación del robot por parte del equipo de trabajo del proyecto.

Si bien el desarrollo de este robot se centra en aplicaciones de tipo académicas, no quedan de lado otras áreas tales como la domótica, agricultura, seguridad, actividades de índole social o a su vez la de exploración y rescate de víctimas, en donde con pequeños ajustes el robot puede trabajar sin problema alguno.

Los nuevos sistemas robóticos cada vez más complejos y costosos, han generado en nuestro entorno como estudiantes o profesionales, una 
idea herrada, obligándonos a pensar que no somos capaces de ser diseñadores y constructores de nuevos y modernos sistemas robóticos y todo por el simple hecho de no disponer de algunos de los dispositivos o lo costoso que puede resultar aquello. Es por eso que se ha tratado de cambiar cada una de estas ideas cerradas con el empleo de elementos de fácil acceso en el medio local dándoles una función diferente e innovadora hasta conseguir la construcción del producto final. El desarrollo de este tipo de sistemas promueve un interés no solo en la construcción sino también en la invención de nuevos y mejorados sistemas que estén en la capacidad de competir en el mercado nacional e internacional.

\section{Referencias}

[1] D. de Informática, "Definición de Robot," Junio - 2012. [Online]. Disponible en: http://www.alegsa.com.ar/Dic/robot.php

[2] "Definición del Robot Industrial ," Junio - 2012. [Online]. Disponible en: http://cfievalladolid2.net/tecno/cyr_ 01/robotica/industrial.htm

[3] "Característica de los Robots ," Junio - 2012. [Online]. Disponible en: http://informaticafrida.blogspot.com/ 2009/03/caracteristica-de-los-robots.html 